ORIGINAL ARTICLE

\title{
Motion resistant pulse oximetry in neonates
}

\author{
R Sahni, A Gupta, K Ohira-Kist, T S Rosen
}

Arch Dis Child Fetal Neonatal Ed 2003;88:F505-F508

See end of article for authors' affiliations .....................

Correspondence to: Dr Sahni, Department of Pediatrics, College of Physicians and Surgeons, Columbia University, 630 W 168th Street, New York, NY 10032, USA; rs62@columbia.edu

Accepted 19 December 2002

\begin{abstract}
Background: Pulse oximetry is widely used in neonates. However, its reliability is often affected by motion artefact. Clinicians confronted with questionable oxygen saturation $\left(\mathrm{SpO}_{2}\right)$ values often estimate the reliability by correlating heart rate (HR) obtained with the oximeter with that obtained by electrocardiogram.

Objective: To compare the effects of motion on $\mathrm{SpO}_{2}$ and $\mathrm{HR}$ measurements made with Masimo signal extraction technology and those made with a Nellcor N-200.

Design: Continuous pulse oximetry and HR monitoring were performed in 15 healthy, term infants (mean (SD) birth weight 3408 (458) g) undergoing circumcision, using Masimo and Nellcor pulse oximeters and a standard HR monitor (Hewlett-Packard). Simultaneous minute by minute behavioural activity codes were also assigned. Baseline data were collected for 10 minutes when the infant was quietly asleep and then continued during and after circumcision for a total duration of one hour. The oximeter $\mathrm{HR}$ and $\mathrm{SpO}_{2}$ values were compared and related to HR values obtained by ECG during all three periods. The effect of behavioural activity on $\mathrm{SpO}_{2}$ and $\mathrm{HR}$ was also evaluated.

Results: When compared with results obtained with the Nellcor, the mean $\mathrm{SpO}_{2}$ and $\mathrm{HR}$ were higher and the incidence of artefact lower with the Masimo during all three periods. Masimo HR more accurately predicted HR obtained with a standard monitor, with lower residual error. $\mathrm{SpO}_{2}$ and $\mathrm{HR}$ values obtained with the Nellcor were lower and more variable during all behavioural states, especially crying, when excessive motion artefact was most likely.

Conclusions: The data suggest that Masimo signal extraction technology may offer improvement in pulse oximetry performance, particularly in clinical situations in which extreme motion artefacts are likely.
\end{abstract}

$P$ ulse oximetry is now the standard non-invasive method of measuring blood oxygenation in neonates. Pulse oxygen saturation $\left(\mathrm{SpO}_{2}\right)$ is measured so commonly that it is now generally accepted as the fifth vital sign. ${ }^{1}$ Although trials have shown that $\mathrm{SpO}_{2}$ measurements obtained by conventional pulse oximetry correlate very closely with blood oxygen saturation ${ }^{2}$ and quite accurately predict $\mathrm{PaO}_{2}{ }^{3}$ they may be affected by subject motion. ${ }^{4}$

Motion artefact limits accurate assessment of oxygenation and may lead to inappropriate responses by clinicians, including both overtreatment and undertreatment. ${ }^{5-8}$ Various strategies have evolved to adjust measurements for presumed motion artefact, such as freezing the display on values thought to be correct or reporting zero. ${ }^{9-11}$ These methods produce misleading information. Faced with suspicious measurement, clinicians usually wait for the motion to stop and then compare the heart rate (HR) value obtained with the oximeter with that obtained by electrocardiogram (ECG). If the two agree, the assumption is that the data are reliable. These periods of absent or inaccurate $\mathrm{SpO}_{2}$ values resulting from motion artefact are not insignificant. Recent reports have shown that movement affects up to $50 \%$ of the recorded tracings in preterm and term infants. ${ }^{12}$

Reduction in the number of false alarms and provision of reliable oximeter readings, even in the presence of patient motion, are two major goals for pulse oximetry in intensive care. Conventional pulse oximetry, based on the ratio of the absorbancy patterns of red and infrared light, is unable to correct the interference caused by patient motion and low perfusion. The Masimo Corporation (Irvine, California, USA) has developed a new electronic signal processing technique and sensor design, called signal extraction technology, which is said to improve the accuracy and reliability of oximetry by superior handling of motion artefact. $^{813-15}$ Although the
Masimo signal extraction technology has performed well in studies using healthy volunteers, evaluations in clinical settings, especially in neonates, have not been reported. The objective of this study was to compare the effects of motion on measurements of $\mathrm{Spo}_{2}$ and HR made with Masimo signal extraction technology, with the same measurements made with the Nellcor N-200.

\section{METHODS}

\section{Study population}

The study population consisted of 15 healthy, term male infants (mean (SD) birth weight 3408 (458) g), all of whom were participants in a randomised controlled study of impact of non-invasive analgesia on the response of the infant to circumcision. All studies were approved by institutional review board, and written consent was obtained from parents of all infants.

\section{Experimental design}

Continuous pulse oximetry and HR monitoring were performed using Masimo signal extraction technology and Nellcor N-200 pulse oximeters and a standard HR monitor (Hewlett-Packard 3680). Baseline data were collected for 10 minutes with the infant quietly asleep. Data collection was then continued during and after circumcision for a total duration of one hour. Simultaneous minute by minute assessments of behavioural sleep and activity state were also made.

Abbreviations: $\mathrm{HR}$, heart rate; $\mathrm{ECG}$, electrocardiogram; $\mathrm{SpO}_{2}$, oxygen saturation 


\section{Experimental method}

All circumcisions were performed in the infant physiology laboratory, located adjacent to the nursery. Infants were brought to the laboratory approximately half an hour before the procedure, at which time ECG leads were attached, and disposable adhesive pulse oximeter sensors (Nellcor N-25 and Masimo low noise optical probe Neo) were placed on the left foot. These sensors were optically shielded from each other to eliminate sensor to sensor optical cross talk. The probes were then connected to the corresponding oximeter. Baseline data were collected for 10 minutes when the infant was quietly asleep with minimal movements. Infants were then given the appropriate analgesia, restrained in the Circumstraint (Olympic Medical, Seattle, Washington, USA), and circumcision was performed. Data collection continued during and after the procedure for the total duration of one hour. Sleep state was coded during the entire study period. Behaviour codes were assigned each minute using a scoring system developed and validated in our laboratory. Active sleep was coded whenever at least one rapid eye movement was observed during the minute, and quiet sleep was designated when the infant was asleep without rapid eye movements. During quiet sleep the infant was relaxed and appeared "ragdoll" floppy with movements limited to startles and jaw jerks. An indeterminate state was coded when small body movements were observed, but no rapid eye movements were seen. Codes were also assigned for wakefulness and crying. ${ }^{16}$

All pulse oximeter data were collected electronically using an RS-232 serial communication port on each instrument. Both $\mathrm{SpO}_{2}$ and the instantaneous HR from each oximeter were recorded digitally once per second throughout the entire study. The ECG was obtained from a standard HR monitor and fed to a special purpose R-R interval preprocessor, where the R-R intervals were calculated with an accuracy of one millisecond. Mean HR for each minute of the data was computed as 60 times the inverse of the mean of the R-R intervals.

\section{Data analysis}

There were times when the oximeter reported no data because of extensive artefact. These epochs were assigned a numeric value of zero by the oximeter rather than a code for no data and no number was displayed. In epochs of less extensive artefact, however, the instruments put out a number that is a moving average of good and artefactual readings. Inclusion of such points designated as zero obviously would amplify the differences between the two oximeters inappropriately, so these measurements were excluded and a different criterion for designation of artefact was developed.

Average HR variability was first computed as the standard deviation of the R-R intervals obtained from the HewlettPackard cardiac monitor for all three periods: baseline, during circumcision, and after circumcision. This normal HR variability ranged from 10.3 to 17.5 beats/min across all infants during the three periods (baseline, 10.3; circumcision, 17.5; after circumcision, 13.7). All oximeter measurements differing from the corresponding ECG measurements by more than 10 beats were designated as artefact. The percentage of epochs designated as artefact was then computed from these edited data for all three periods. Minute averages for $\mathrm{Spo}_{2}$ and HR were also computed from the artefact-free oximeter data. Behavioural state codes were logged to the appropriate minutes. The amount of artefact in the raw data from the two instruments, expressed as percentage artefact, was compared for all three periods using $t$ tests. After removal of the unreported artefact, as described above, the $\mathrm{SpO}_{2}$ and HR readings of the two instruments were compared, also using $t$ tests. Further, simple linear regression was used to compare the artefact-free HR data obtained from the Masimo and Nellcor oximeters with the reference standard HR obtained from the Hewlett-Packard cardiac monitor during all three periods. The effect of behavioural activity on $\mathrm{SpO}_{2}$ and HR data was determined separately for each behavioural state.

\section{RESULTS}

The incidence of artefact (with the above defined artefact criteria) was significantly lower with the Masimo than with the Nellcor during (mean (SD) percentage artefact: 25.2 (5.9) $v 63.8$ (5.8), $\mathrm{p}<0.00001)$ and after (7.7 (2.2) $v 33.8$ (4.9), $\mathrm{p}<0.0001)$ circumcision. Even during the baseline period when the infants were quietly asleep, there was a threefold higher incidence of artefact with the Nellcor (4.0 (1.9) $v 13.1$ (14.9), $\mathrm{p}=0.06$; table 1 ).

Mean (SD) $\mathrm{Spo}_{2}$ and HR values obtained from the Masimo were significantly higher than those obtained with the Nellcor, especially during circumcision, when extreme motion was likely (96.9 (0.4) v 92.8 (1.0), $\mathrm{p}<0.0005$, and $151.5(4.5) \vee 133.6(5.6), \mathrm{p}<0.001$ respectively; table 2). Similar differences were observed after circumcision.

Regression analysis of HR measurements from both oximeters and ECG HR revealed that the Masimo HR signal predicted the ECG HR more accurately $\left(r^{2}=0.72-0.86 v\right.$ $0.28-0.63)$, with lower residual error ( $1.3-5.8$ v 3.9-19.5 beats/ min), during all three periods (table 3 ).

As shown in figs 1 and 2, the performance of the two oximeters varied, with different behavioural activity states during the study period. The $\mathrm{HR}$ and $\mathrm{SpO}_{2}$ measured by Nellcor were lower and more variable during all behavioural states. The effects were most pronounced during behavioural activity states associated with excessive motion artefactthat is, active sleep, awake, and crying. As expected, during non-sleeping states (awake, crying), higher HR values were recorded by the Masimo; however, the Nellcor recorded not only much lower but more variable HR values during active sleep, awake, and crying states than those recorded during quiet sleep. This was also reflected in the $\mathrm{Spo}_{2}$ measurements, which remained stable for Masimo but were significantly lower for Nellcor during all behavioural activity states. For example, during crying $(64 \%$ of the crying behaviour was observed during the circumcision period), when extreme motion artefact was most likely, the Nellcor recorded significantly lower $\mathrm{Spo}_{2}$ than the Masimo (mean (SD) 92.5 (9.9) $v 95.2(4.2), \mathrm{p}<0.0005)$.

\section{DISCUSSION}

The accuracy and reliability of conventional pulse oximetry is limited by subject motion. ${ }^{4}$ A possible explanation for this limitation involves the confounding effects of venous blood flow. Conventional pulse oximeters assume that the arterial compartment is the only compartment that contains pulsating blood flow. During patient motion, however, the venous

Table 1 Mean percentage artefact (defined as all pulse oximetry data with a difference greater than 10 beats between the pulse oximeter heart rate and the corresponding ECG heart rate) with Masimo and Nellcor pulse oximeters before, during, and after circumcision $(n=15)$

\begin{tabular}{lccc}
\hline & Masimo & Nellcor & p Value \\
\hline Baseline & $4.0(1.9)$ & $13.1(14.9)$ & 0.06 \\
Circumcision & $25.2(5.9)$ & $63.8(5.8)$ & $<0.00001$ \\
After & $7.7(2.2)$ & $33.8(4.9)$ & $<0.0001$ \\
\hline
\end{tabular}

Values are mean (SD). 
Table 2 Oxygen saturation $\left(\mathrm{SpO}_{2}\right)$ and heart rate $(\mathrm{HR})$ recorded with Masimo and Nellcor pulse oximeters (artefact-free data) before, during, and after circumcision $(n=15)$

\begin{tabular}{|c|c|c|c|c|c|c|c|c|c|}
\hline & \multicolumn{3}{|l|}{ Baseline } & \multicolumn{3}{|l|}{ Circumcision } & \multicolumn{3}{|c|}{ After circumcision } \\
\hline & Masimo & Nellcor & p Value & Masimo & Nellcor & $\mathrm{p}$ Value & Masimo & Nellcor & $\mathrm{p}$ Value \\
\hline $\begin{array}{l}\mathrm{SpO}_{2}(\%) \\
\mathrm{HR} \text { (beats/ } \\
\text { min) }\end{array}$ & $\begin{array}{l}98.2(0.3) \\
126.6(3.0)\end{array}$ & $\begin{array}{l}97.5(0.3) \\
123.2(3.6)\end{array}$ & $\begin{array}{l}\text { NS } \\
0.04\end{array}$ & $\begin{array}{l}96.9(0.4) \\
151.5(4.5)\end{array}$ & $\begin{array}{l}92.8(1.0) \\
133.6(5.6)\end{array}$ & $\begin{array}{l}<0.0005 \\
<0.001\end{array}$ & $\begin{array}{l}98.1(0.2) \\
132.6(3.5)\end{array}$ & $\begin{array}{l}95.6(1.2) \\
127.2(3.5)\end{array}$ & $\begin{array}{l}<0.03 \\
<0.0001\end{array}$ \\
\hline
\end{tabular}

blood also moves irregularly, which adds a confounding signal to the pulse waveform signal, and thereby changes the apparent amount of light transmitted to the photoreceptor at the two wavelengths, red and infrared. These are the two wavelengths that are used to detect the relative proportions of oxyhaemoglobin and deoxyhaemoglobin in the tissue (red/ infrared transmitted light ratio). ${ }^{17-19}$ The magnitude of error in $\mathrm{SpO}_{2}$ measurement will be influenced by venous blood saturation, arterial signal amplitude, and magnitude of motion. ${ }^{49}$ The motion-added signal produces false $\mathrm{SpO}_{2}$ values to the extent that the extra signal, which does not represent the arterial pulse oxyhaemoglobin/deoxyhaemoglobin signal, is large relative to the arterial pulse signal. When the motion is major, absolutely or in relation to arterial pulse signal, the motion-added signal tends to predominate over the pulse signal, so that the red/infrared ratio transmitted to the photoreceptor produces a false $\mathrm{SpO}_{2}$ value. An additional and specific source of error in conventional pulse oximetry occurs when noise from a variety of signal inputs that are not a part of the true arterial pulse signal is introduced equally into both the red and infrared channels, driving the red/ infrared ratio towards unity. This ratio of 1.0 predicts a saturation of $\sim 82 \%$ according to conventional pulse oximetry algorithm. ${ }^{4}{ }^{19}$ Such motion and noise artefacts are disturbing. They also limit accurate assessment of oxygenation, particularly in the most ill patients, who are most susceptible to erroneous saturation readings.

Periods of absent or inaccurate $\mathrm{Spo}_{2}$ values due to motion artefact are not insignificant, and may affect up to $50 \%$ of the recorded tracings in preterm and term infants. ${ }^{12}$ Motion artefact during pulse oximetry recordings is also dependent on behavioural state. Recent reports have documented as many as six changes in behavioural state per hour in preterm infants, with at least 10 changes that last more than 10 minutes per hour. ${ }^{12}$ Nursing interventions and noise independently result in significant changes in both the behavioural and physiological responses of infants studied..$^{20}$ Such problems are worse during transport and during more physically active periods such as resuscitations. ${ }^{21-22}$ These are specific situations in which accurate pulse oximetry values would improve our understanding of abnormal oxygenation and the effects of oxygen therapy and other interventions.

Various methods have been devised to minimise the loss of signal associated with motion. Most of these involve averaging the signal over longer periods of time and/or rejecting rapid unphysiological changes in the signal ratio. ${ }^{11}$ Both techniques represent best guesses rather than true measurements. A new electronic signal processing technique and sensor design, called signal extraction technology by Masimo Corporation, has been reported to considerably decrease false $\mathrm{SpO}_{2}$ values during patient motion. ${ }^{813} 1523$ The Masimo paradigm is based on the assumption that arterial blood is not the only absorptive substance in tissue that may generate a pulsatile optical signal. An adaptive filter system is used to separately identify the energy present at each saturation in the range $1-100 \%$ in $0.5 \%$ increments every 0.4 second. Once the discrete saturation transformation identifies and verifies the energy peak produced by the pulsating arterial blood, which is usually the peak corresponding to the highest saturation in the discrete saturation transformation, it reports this value as the patient's $\mathrm{SpO}_{2}$. Thus, it is able to cancel the noise signal and separate the arterial signals from artefacts. This results in superior signal acquisition and reduces the risk of false alarms during motion at the monitoring site. ${ }^{19}$

Our study shows that the $\mathrm{SpO}_{2}$ and $\mathrm{HR}$ signals from Masimo are less variable, and the Masimo HR signal more accurately predicts the Hewlett-Packard HR during both resting and increased activity states. With signal extraction technology, the superior signal handling during increased activity states associated with higher degree of motion artefact is understandable, but differences are also observed during baseline and behavioural quiet sleep periods. Various factors could potentially contribute to these differences. The signal drop out rate-that is, periods with no acceptable data-is much higher with conventional oximetry and may contribute most significantly to these differences. Of course, the internal smoothing algorithms and other editing functions limit the impact of these disturbances on the digital output of both devices, and the zero outputs are invisible to the observer. For example, in our study, during quiet sleep, which is associated with greatly reduced but not totally absent physical activity, the drop out rate in the raw data was 50 times greater for the Nellcor than the Masimo oximeter $(4.81 \% v 0.09 \%)$. An artefact reduction algorithm was applied to the raw data before the comparative analysis, and, despite that, the performance of the Masimo oximeter was noted to be superior. Another possible factor that may have contributed to these differences is sensor to sensor cross talk, as

Table 3 Relation of Masimo and Nellcor oximeter heart rates to cardiac monitor heart rate before, during, and after circumcision $(n=15)$

\begin{tabular}{|c|c|c|c|c|c|c|}
\hline & \multicolumn{2}{|l|}{ Baseline } & \multicolumn{2}{|c|}{ Circumcision } & \multicolumn{2}{|c|}{ After circumcision } \\
\hline & Masimo & Nellcor & Masimo & Nellcor & Masimo & Nellcor \\
\hline $\begin{array}{l}\text { Correlation coefficient }\left(r^{2}\right) \\
\text { Residual error (beats } / \mathrm{min})\end{array}$ & $\begin{array}{l}0.86 \\
1.3\end{array}$ & $\begin{array}{l}0.63 \\
3.9\end{array}$ & $\begin{array}{l}0.72 \\
5.8\end{array}$ & $\begin{array}{l}0.28 \\
19.5\end{array}$ & $\begin{array}{l}0.78 \\
2.4\end{array}$ & $\begin{array}{l}0.31 \\
14.6\end{array}$ \\
\hline
\end{tabular}




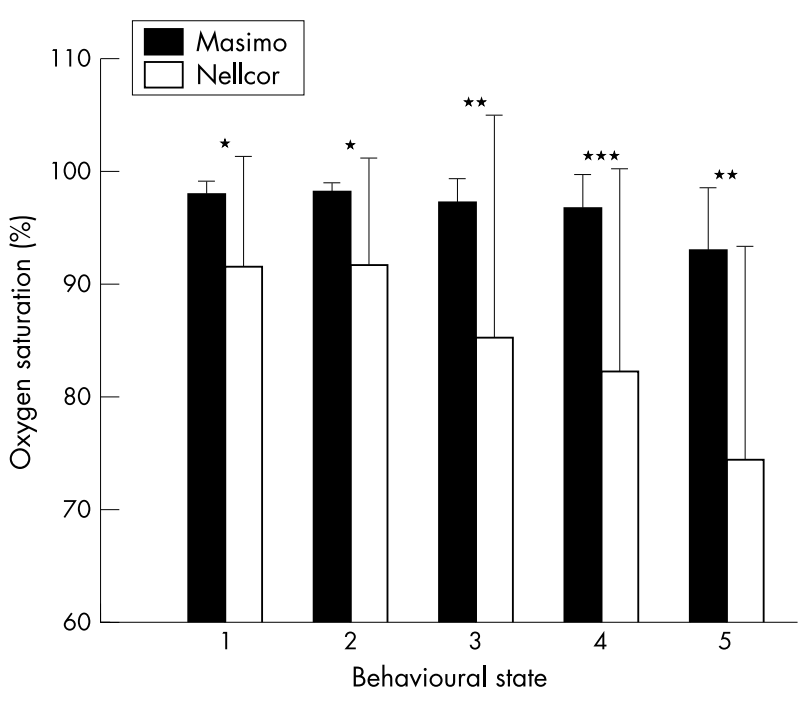

Figure 1 Oxygen saturation measurements (means (SD)) with Masimo and Nellcor pulse oximeters during different behavioural activity states: 1, quiet sleep; 2 , indeterminate sleep; 3 , active sleep; 4, awake; 5 , crying. ${ }^{*} \mathrm{p}<0.02 ;{ }^{* *} \mathrm{p}<0.005 ;{ }^{* * *} \mathrm{p}<0.0005$.

both oximeter sensors were on the same foot. During the planning of this experimental protocol, we had noted that the leg movements were not symmetrical when the infants were restrained in the Circumstraint, particularly during times of excessive motion (circumcision). This movement asymmetry would have significantly affected our primary objective of comparing the oximeter performance during motion. So, to test the oximeter performance under a similar degree of motion, the sensors were placed on the dorsum of the left foot as far from each other as possible and optically shielded to prevent cross talk. However, this may not have completely eliminated it, and cross talk may have contributed to minor differences in $\mathrm{Spo}_{2}$; this is a study limitation.

Our data suggest that the Masimo signal extraction technology may improve pulse oximetry performance particularly in clinical situations in which extreme motion is likely, such as circumcision and crying in our neonates. Also, superior performance during times of minimal motion or when the infant is motionless suggests better signal acquisition and processing with the Masimo signal extraction technology. Further studies investigating the effects of this new technology on clinical care and outcomes will be necessary. Such application studies should include evaluation of nursing time and effort, medical judgments about routine oxygen deficits and oxygen therapy, and assessments of usefulness during active behaviour, transport, and resuscitation. Instrumentation that is considerably more accurate and reliable, with much greater sensitivity and specificity, may offer a definite advantage over current home monitoring systems, which have notoriously high rates of false alarms. A more accurate and reliable pulse oximeter should have the capacity to refine the therapeutic use of oxygen and benefit patient management and outcome.

\section{Authors' affiliations}

R Sahni, A Gupta, K Ohira-Kist, T S Rosen, College of Physicians and Surgeons, Columbia University, New York, NY, USA

Presented at the Pediatric Academic Societies and the American Academy of Pediatrics Joint Meeting, May 12-16, 2000, Boston, MA, USA.

This study received equipment support and technical assistance from Masimo Corporation, Irvine, California, USA.

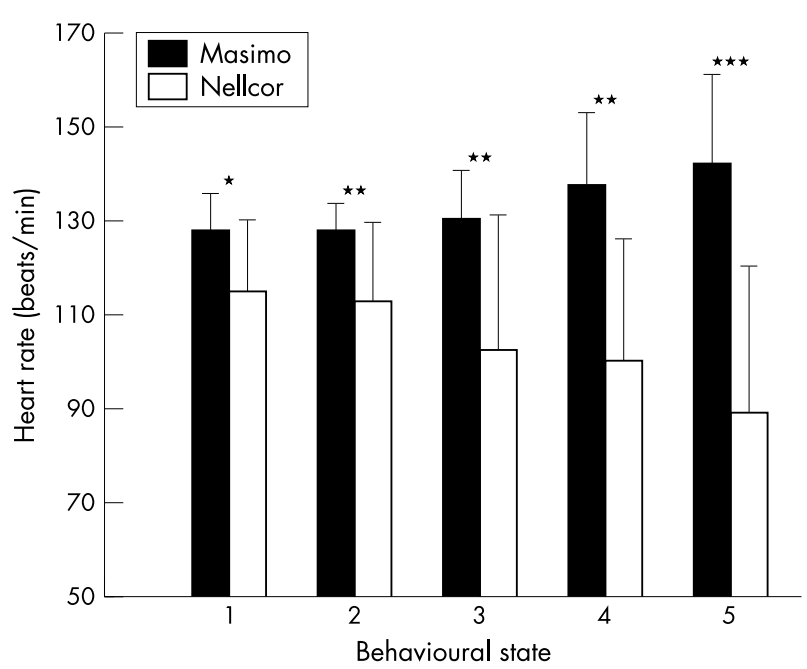

Figure 2 Heart rate measurement (means (SD)) with Masimo and Nellcor pulse oximeters during different behavioural activity states: 1 , quiet sleep; 2 , indeterminate sleep; 3 , active sleep; 4 , awake; 5 , crying. ${ }^{*} \mathrm{p}<0.005 ;{ }^{* *} \mathrm{p}<0.0005 ;{ }^{* * *} \mathrm{p}<0.00005$.

\section{REFERENCES}

1 Neff TA. Routine oximetry: a fifth vital sign? Chest 1998;94:227.

2 Hay WW Jr, Brockway J, Eyzaguirre M. Neonatal pulse oximetry: accuracy and reliability. Pediatrics 1989;83:717-22.

3 Brockway J, Hay WW Jr. Prediction of arterial partial pressure of oxygen $\left(\mathrm{PaO}_{2}\right)$ with pulse oxygen saturation $\left(\mathrm{SpO}_{2}\right)$ measurements. J Pediatr $1998 ; 133: 63-6$.

4 Barker SJ, Shah NK. The effects of motion on the performance of pulse oximeter in volunteers. Anesthesiology 1997:86:101-8.

5 Sabar R, Zmora E. Nurses' response to alarms from monitoring systems in NICU [abstract]. Pediatr Res 1997;41:174A

6 Lawless ST. Crying wolf: false alarms in a pediatric intensive care unit. Crit Care Med 1994;22:981-5.

7 Stoneham MD, Saville GM, Wilson $\mathrm{IH}$. Knowledge about pulse oximetry among medical and nursing staff. Lancet 1994;344:1339-42.

8 Goldstein MR, Martin GI, Sindel BD, et al. Novel pulse oximeter technology resistant to noise artefact and low perfusion [abstract]. Am J Respir Crit Care Med 1997; 155:A712.

9 Rheineck-Leyssius AT, Kalkman CJ. Influence of pulse oximetry settings on the frequency of alarms and detection of hypoxemia: theoretical effects of artefact rejection, alarm delay, averaging, median filtering, or a lower setting of alarm limit. Int J Clin Monit Comput 1998;14:151-6.

10 Nellcor PB. Technology overview: $\mathrm{SpO}_{2}$ monitors and Oxismart. Note internal code no: 9:00819-0698.

11 Pan PH James CF. Effects of default alarm settings on alarm distribution in telemetric pulse oximetry network in ward setting [abstract]. Anesthesiology 1991;75:A405

12 Fletcher J, Page $M$, Jeffery HE. Sleep states and neonatal pulse oximetry Sleep 1998:21:305-10.

13 Dumas C, Wahr JA, Tremper KK. Clinical evaluation of a prototype motion artefact resistant pulse oximeter in recovery room. Anesth Analg 1996:83:269-72

14 Barnum PT, Taschuk RD, Goldstein MR, et al. Novel pulse oximetry technology capable of reliable bradycardia monitoring in the neonate [abstract]. Respir Care 1997;42:1072A.

15 Bohnhorst B, Poets CF. Major reduction in alarm frequency with a new pulse oximeter. Intensive Care Med 1998:24:277-8.

16 Stefanski M, Schulze KF, Bateman D, et al. A scoring system for states of sleep and wakefulness in term and preterm infants. Pediatr Res 1984;18:58-63.

17 Hay WW Jr. Physiology of oxygenation and its relation to pulse oximetry in neonates. J Perinatol 1987:7:309-19.

18 Hay WW, Thilo E, Curlander JB. Pulse oximetry in neonatal medicine. Clin Perinatol 1991; 18:441-72.

19 Goldman JM, Petterson MT, Kopotic RJ, et al. Masimo Signal Extraction Pulse Oximetry. J Clin Monit 2000;16:475-483.

20 Zahr LK, Balian S. Responses of premature infants to routine nursing interventions and noise in the NICU. Nurs Res 1995;44:179-85.

21 Goldstein MR, Liberman RI, Taschuk RD, et al. Pulse oximetry in transport of poorly perfused babies. Pediatrics 1998;2:818.

22 House JT, Schultetus RR, Gravenstien N. Continuous neonatal evaluation in the delivery room by pulse oximetry. Int J Clin Monit Comput 1998; 14:49-55.

23 Hay WW Jr, Rodden DJ, Collins SM, et al. Pulse oximetry in NICU: conventional vs. Masimo SET [abstract]. Pediatr Res 1999;45:304A 